Henryk Ko€AKowski and JarosŁaw Łazuka (Warszawa)

\title{
ON THE THEORY OF THERMOELASTICITY
}

Abstract. The aim of this paper is to prove some properties of the solution to the Cauchy problem for the system of partial differential equations describing thermoelasticity of nonsimple materials proposed by D. Iesan. Explicit formulas for the Fourier transform and some estimates in Sobolev spaces for the solution of the Cauchy problem are proved.

In this paper we consider the following initial value problem:

$$
\begin{gathered}
\begin{aligned}
& u_{t t}=c_{2}^{2}\left(1-l_{2}^{2} \Delta\right) \Delta u+\left[c_{1}^{2}-c_{2}^{2}-\left(c_{1}^{2} l_{1}^{2}-\right.\right.\left.\left.c_{2}^{2} l_{2}^{2}\right) \Delta\right] \nabla \operatorname{div} u \\
&-\frac{m}{\rho} \nabla\left(\theta+\alpha \theta_{t}\right)+f, \\
& k \Delta \theta-c\left(\theta_{t}+\beta \theta_{t t}\right)-T_{0} m \operatorname{div} u_{t}= \rho s \quad \text { for } t>0, \\
& u(0, x)=u^{0}(x), \quad u_{t}(0, x)=u^{1}(x), \quad \theta(0, x)=\theta_{0}(x), \quad \theta_{t}(0, x)=\theta_{1},
\end{aligned}
\end{gathered}
$$

where $x \in \mathbb{R}^{3}, u: \overline{\mathbb{R}}_{+} \times \mathbb{R}^{3} \rightarrow \mathbb{R}^{3}, \theta: \overline{\mathbb{R}}_{+} \times \mathbb{R}^{3} \rightarrow \mathbb{R}, u$ denotes the displacement, $\theta$ the temperature disturbance, $f$ is a given vector-valued function on $[0, \infty) \times \mathbb{R}^{3}$ and $s$ is a given function also on $[0, \infty) \times \mathbb{R}^{3} ; c_{1}, c_{2}, l_{1}, l_{2}, m, k, c, \rho$, $T_{0}, \alpha, \beta$ are constant physical parameters.

In [4] we considered the Cauchy problem for the system of equations describing thermoelasticity of nonsimple materials proposed by G. Ahmadi and K. Firoozbakhsh [1]. This theory of thermoelasticity is based on the Clausius-Duhem inequality and it is assumed that the potential energy density depends on the strain, the gradient of the strain and the temperature. The theory of thermoelasticity of nonsimple materials proposed by D. Iesan [3] is different from the theory of G. Ahmadi and K. Firoozbakhsh [1]. In Iesan's theory the local state is characterized by the deformation gradient, its gradient, the temperature, its gradient, and the time rate of change of temperature. Iesan [3] obtained restrictions on constitutive equations with the

2010 Mathematics Subject Classification: Primary 35Q79.

Key words and phrases: Cauchy problem, thermoelasticity, Sobolev spaces. 
help of an entropy production inequality proposed by Green and Laws [2] Using conservation laws and constitutive equations Iesan [3] derived the system (1). These equations are different from the equations of Ahmadi and Firoozbakhsh [4], [1. The differences between those two systems are significant, i.e. the equation of energy balance contains the second order derivative of the temperature with respect to time and the equations of motions contain a term with the gradient of the first order derivative of the temperature with respect to time.

The main results of this paper are an exact formula for the solution $(u, \theta)$ of the Cauchy problem (1)-(2) and the following theorem:

TheOREM 1. Let $u^{0}, \theta_{0}, \theta_{1}$ be in the Sobolev space $H^{s+1}\left(\mathbb{R}^{3}\right)$ with norm $\|\cdot\|_{s+1}, u^{1} \in H^{s}\left(\mathbb{R}^{3}\right), f \in C^{2}\left([0, t], H^{s+1}\left(\mathbb{R}^{3}\right)\right), \mathbf{s} \in C^{2}\left([0, t], H^{s+1}\left(\mathbb{R}^{3}\right)\right)$ and $\alpha>\beta$. Then the problem (1)-(2) has a unique solution $u, \theta \in C^{2}([0, t]$, $\left.H^{s}\left(\mathbb{R}^{3}\right)\right)$. This solution satisfies, for some constant $C$, the estimates

$$
\begin{aligned}
\|u\|_{s}^{2} \leq & C\left(1+t^{4}\right)\left(\left\|u^{0}\right\|_{s}^{2}+\left\|u^{1}\right\|_{s}^{2}+\left\|\theta_{0}\right\|_{s+1}^{2}+\left\|\theta_{1}\right\|_{s+1}^{2}\right. \\
& \left.+\sup _{\tau \in[0, t]}\|f(\tau)\|_{s}^{2}+\sup _{\tau \in[0, t]}\|\mathbf{s}\|_{s+1}^{2}\right), \\
\|\theta\|_{s}^{2} \leq & C\left(1+t^{4}\right)\left(\left\|u^{0}\right\|_{s+1}^{2}\left\|u^{1}\right\|_{s}^{2}+\left\|\theta_{0}\right\|_{s}^{2}+\left\|\theta_{1}\right\|_{s}^{2}\right. \\
& \left.+\sup _{\tau \in[0, t]}\|f(\tau)\|_{s-1}^{2}+\sup _{\tau \in[0, t]}\|\mathbf{s}\|_{s+1}^{2}\right) .
\end{aligned}
$$

Now we derive the formulae for the Fourier transform of the solution to the Cauchy problem (1)-(2). Let $u_{t}=v, \theta_{t}=\eta$. Then the system (1) can be rewritten in the form

$$
\begin{aligned}
& u_{t}=v, \\
& v_{t}=c_{2}^{2}\left(1-l_{2}^{2} \Delta\right) \Delta u+\left[c_{1}^{2}-c_{2}^{2}-\left(c_{1}^{2} l_{1}^{2}-c_{2}^{2} l_{2}^{2}\right) \Delta\right] \nabla \operatorname{div} u-\frac{m}{\rho} \nabla(\theta+\alpha \eta)+f, \\
& \theta_{t}=\eta, \\
& \eta_{t}=\frac{1}{c \beta}\left(-T_{0} m \operatorname{div} v-c \eta+k \Delta \theta-\rho s\right),
\end{aligned}
$$

or briefly

$$
V_{t}=P\left(D_{x}\right) V+F
$$

where

$$
D_{x_{j}}=\frac{1}{i} \frac{\partial}{\partial x_{j}}, \quad V=[u, v, \theta, \eta]^{T}, \quad F=[0, f, 0,-\rho s / c \beta]^{T} .
$$

We denote by $P\left(D_{x}\right)$ the differential operator with symbol $P(\xi)$. It can be 
shown that

$$
\lambda I-P(\xi)=\left|\begin{array}{cccccccc}
\lambda & 0 & 0 & -1 & 0 & 0 & 0 & 0 \\
0 & \lambda & 0 & 0 & -1 & 0 & 0 & 0 \\
0 & 0 & \lambda & 0 & 0 & -1 & 0 & 0 \\
a_{11} & a_{12} & a_{13} & \lambda & 0 & 0 & \tilde{\beta} \xi_{1} & \alpha \tilde{\beta} \xi_{1} \\
a_{21} & a_{22} & a_{23} & 0 & \lambda & 0 & \tilde{\beta} \xi_{2} & \alpha \tilde{\beta} \xi_{2} \\
a_{31} & a_{32} & a_{33} & 0 & 0 \lambda & 0 & \tilde{\beta} \xi_{3} & \alpha \tilde{\beta} \xi_{3} \\
0 & 0 & 0 & 0 & 0 & 0 & \lambda & -1 \\
0 & 0 & 0 & \tilde{\alpha} \xi_{1} & \tilde{\alpha} \xi_{2} & \tilde{\alpha}_{3} & D & \lambda+1 / \beta
\end{array}\right|
$$

where

$$
\begin{gathered}
\tilde{\beta}=i \frac{m}{\rho}, \tilde{\alpha}=i \frac{T_{0} m}{c^{2} \beta}, \quad D=\frac{k|\xi|^{2}}{c \beta}, \quad\left\{a_{i j}\right\}=a_{2} I+b \xi \otimes \xi \\
\left.a_{k}=c_{k}^{2} l_{k}^{2}|\xi|^{4}+c_{k}^{2}|\xi|^{2}, \quad b=\left[c_{1}^{2} l_{1}^{2}-c_{2}^{2} l_{2}^{2}\right)|\xi|^{2}+\left(c_{1}^{2}-c_{2}^{2}\right)\right] .
\end{gathered}
$$

The characteristic polynomial of the matrix $P(\xi)$ has the form

$$
\operatorname{det}[\lambda I-P(\xi)]=\left(\lambda^{2}+a_{2}\right)^{2} W(\lambda),
$$

where

$$
\begin{aligned}
W(\lambda)= & \lambda^{4}+\frac{1}{\beta} \lambda^{3}+\frac{1}{c \beta}\left(a_{1} c \beta+k|\xi|^{2}+\frac{T_{0} m^{2}}{\rho} \alpha|\xi|^{2}\right) \lambda^{2} \\
& +\frac{1}{c \beta}\left(\frac{T_{0} m^{2}}{\rho}|\xi|^{2}+a_{1} c\right) \lambda+\frac{k|\xi|^{2} a_{1}}{c \beta} .
\end{aligned}
$$

This fact is a consequence of further calculations. Let $w=\operatorname{rot} u$. Applying the rotation operator to the first equation of the system (1) we get the equation

$$
w_{t t}+c_{2}^{2} l_{2}^{2} \Delta^{2} w-c_{2}^{2} \Delta w=\operatorname{rot} f
$$

with initial data

$$
w(0, x)=\operatorname{rot} u^{0}(x), \quad w_{t}(0, x)=\operatorname{rot} u^{1}(x) .
$$

Thus for the Fourier transform of $w$ with respect to the spatial variables we obtain the Cauchy problem

$$
\begin{aligned}
\hat{w}_{t t}+a_{2} \hat{w}=\widehat{\operatorname{rot} f} & \text { for } t>0, \\
\hat{w}=\widehat{\operatorname{rot} u^{0}}, \quad \hat{w}_{t}=\widehat{\operatorname{rot} u^{1}} & \text { for } t=0 .
\end{aligned}
$$

The initial-value problem (6) has, for appropriate functions $f, u^{0}, u^{1}$, the unique solution

$$
\hat{w}=\widehat{\operatorname{rot} u^{0}} \cos \sqrt{a_{2}} t+\widehat{\frac{\operatorname{rot} u^{1}}{\sqrt{a_{2}}}} \sin \sqrt{a_{2}} t+\int_{0}^{t} \frac{\sin \left[\sqrt{a_{2}}(t-s)\right] \widehat{\operatorname{rot} f(s)}}{\sqrt{a_{2}}} d s .
$$


Now, applying the divergence operator to the first equation of the system (1) and using the notation $e=\operatorname{div} u$, we obtain the system

$$
\begin{gathered}
e_{t t}-c_{2}^{2} \Delta e+c_{2}^{2} l_{2}^{2} \Delta^{2} e-\left(c_{1}^{2}-c_{2}^{2}\right) \Delta e \\
+\left(c_{1}^{2} l_{1}^{2}-c_{2}^{2} l_{2}^{2}\right) \Delta^{2} e+\frac{m}{\rho} \Delta\left(\theta+\alpha \theta_{t}\right)=\operatorname{div} f, \\
k \Delta \theta-c\left(\theta_{t}+\beta \theta_{t t}\right)-T_{0} m e_{t}=\rho s .
\end{gathered}
$$

Hence

$$
\begin{aligned}
e_{t t}+c_{1}^{2} l_{1}^{2} \Delta^{2} e+\frac{m}{\rho} \Delta\left(\theta+\alpha \theta_{t}\right)-c_{1}^{2} \Delta e & =\operatorname{div} f, \\
k \Delta \theta-c\left(\theta_{t}+\beta \theta_{t t}\right)-T_{0} m e_{t} & =\rho s .
\end{aligned}
$$

Applying the Fourier transform with respect to the spatial variables we get

$$
\begin{aligned}
\hat{e}_{t t}+c_{1}^{2} l_{1}^{2}|\xi|^{4} \hat{e}-\frac{m}{\rho}|\xi|^{2}\left(\hat{\theta}+\alpha \hat{\theta}_{t}\right)+c_{1}^{2}|\xi|^{2} \hat{e} & =\widehat{\operatorname{div} f} \\
-c \beta \hat{\theta}_{t t}-c \hat{\theta}_{t}-k|\xi|^{2} \hat{\theta}-T_{0} m \hat{e}_{t} & =\widehat{\rho s} .
\end{aligned}
$$

Let $\hat{e}_{t}=\hat{v}$. Then

$$
\begin{aligned}
& \hat{e}_{t}=\hat{v}, \\
& \hat{v}_{t}=-\left(c_{1}^{2} l_{1}^{2}|\xi|^{4}+c_{1}^{2}|\xi|^{2}\right)+\frac{m}{\rho}|\xi|^{2}\left(\hat{\theta}+\alpha \hat{\theta}_{t}\right)+\widehat{\operatorname{div} f}, \\
& \hat{\theta}_{t}=\hat{\eta}, \\
& \hat{\eta}_{t}=-\frac{1}{\beta} \hat{\eta}-\frac{k}{c \beta}|\xi|^{2} \hat{\theta}-\frac{T_{0} m}{c \beta} \hat{v}+\frac{\rho}{c \beta} \hat{s} .
\end{aligned}
$$

This system can be expressed in the simple form

$$
Y_{t}=A Y+G,
$$

where $Y=[\hat{e}, \hat{v}, \hat{\theta}, \hat{\eta}]^{T}, G=[0, \widehat{\operatorname{div} f}, 0, \hat{\mathbf{s}}]^{T}, \mathbf{s}=\frac{\rho}{c \beta} s$. It can be easily seen that (see (4))

$$
\operatorname{det}[\lambda I-A]=W(\lambda) .
$$

The polynomial $W(\lambda)$ has four distinct roots $\lambda_{1}, \lambda_{2}, \lambda_{3}, \lambda_{4}$. Note that

$$
\begin{aligned}
-\left(\lambda_{1}+\lambda_{2}+\lambda_{3}+\lambda_{4}\right) & =\frac{1}{\beta} \\
\lambda_{4} \lambda_{3}+\lambda_{4} \lambda_{2}+\lambda_{4} \lambda_{1}+\lambda_{3} \lambda_{2}+\lambda_{3} \lambda_{1} & +\lambda_{2} \lambda_{1} \\
& =a_{1}+\frac{k}{c \beta}|\xi|^{2}+\alpha \frac{m}{\rho}|\xi|^{2} \frac{T_{0} m}{c \beta} .
\end{aligned}
$$

For $\alpha>\beta$ the polynomial $W$ is stable, i.e. all its roots have negative real parts (for $\xi \neq 0$ ). Indeed, according to [5, Theorem 6], if all principal minors 
of the matrix

$$
\left[\begin{array}{cccc}
a_{1} & a_{3} & 0 & 0 \\
a_{0} & a_{2} & a_{4} & 0 \\
0 & a_{1} & a_{3} & 0 \\
0 & a_{0} & a_{2} & a_{4}
\end{array}\right]
$$

are positive, then the polynomial $w(\lambda)=a_{0} \lambda^{4}+a_{1} \lambda^{3}+a_{2} \lambda^{2}+a_{3} \lambda+a_{4}$ with $a_{0}>0$ is stable. Equivalently, if $a_{0}>0, a_{1}>0, a_{2}>0, a_{3}>0, a_{4}>0$, $a_{1} a_{2} a_{3}-a_{0} a_{3}^{2}-a_{0}^{2} a_{4}>0$ then the polynomial $w$ is stable. In our case

$$
a_{1} a_{2} a_{3}-a_{0} a_{3}^{2}-a_{0}^{2} a_{4}=\frac{1}{c^{2} \beta^{2}}\left[\psi^{2}(\alpha-\beta)\left(\psi^{2}+\psi a_{1} c\right)+\chi \psi\right]
$$

where

$$
\psi=\frac{T_{0} m^{2}}{\rho}|\xi|^{2}, \quad \chi=k|\xi|^{2} .
$$

Thus the polynomial $W$ satisfies the stability condition for $\alpha>\beta$.

Putting $G=0$ in the system (8) we obtain the following initial-value problem:

$$
Y_{t}=A Y, \quad Y(0)=\left[\hat{e}_{0}, \hat{v}_{0}, \hat{\theta}_{0}, \hat{\eta}_{0}\right]^{T} .
$$

The solution of this problem is

$$
Y=c_{1} Y_{1}+c_{2} Y_{2}+c_{3} Y_{3}+c_{4} Y_{4}
$$

where

$$
\begin{aligned}
Y_{j}(t) & =\left[\frac{(\tilde{k}+\alpha \tau \tilde{m}) \lambda_{j}+\lambda_{j}^{2} \frac{1}{\beta}+\lambda_{j}^{3}+\tilde{m} \tau}{a_{1} \tilde{m}}, \frac{-\tilde{k}-\frac{1}{\beta} \lambda_{j}-\lambda_{j}^{2}}{\tilde{m}}, 1, \lambda_{j}\right]^{T} e^{\lambda_{j} t} \\
& =:\left[Y_{j 1}, Y_{j 2}, Y_{j 4}, Y_{j 4}\right]^{T},
\end{aligned}
$$

and

$$
\tilde{m}=\frac{T_{0} m}{c \beta}, \quad \tilde{k}=\frac{k}{c \beta}|\xi|^{2}, \quad \tau=\frac{m}{\rho}|\xi|^{2} .
$$

Let $B=\left[Y_{1}(0), Y_{2}(0), Y_{3}(0), Y_{4}(0)\right], c=\left[c_{1}, c_{2}, c_{3}, c_{4}\right]^{T}$. Then

$$
B c=Y(0) \text {. }
$$

Using (9) and (10) gives

$$
\begin{aligned}
\operatorname{det} B= & \frac{\left(\lambda_{2}-\lambda_{1}\right)\left(\lambda_{3}-\lambda_{1}\right)\left(\lambda_{4}-\lambda_{1}\right)\left(\lambda_{3}-\lambda_{2}\right)\left(\lambda_{4}-\lambda_{2}\right)\left(\lambda_{4}-\lambda_{3}\right)}{a_{1} \tilde{m}^{2}}, \\
c_{1}= & \frac{1}{\left(\lambda_{2}-\lambda_{1}\right)\left(\lambda_{3}-\lambda_{1}\right)\left(\lambda_{4}-\lambda_{1}\right)}\left[-a_{1} \tilde{m} \hat{e}_{0}+\lambda_{1} \tilde{m} \hat{v}_{0}\right. \\
& \left.+\left(\tilde{k} \lambda_{1}+\lambda_{2} \lambda_{3} \lambda_{4}+\tilde{m} \tau\right) \hat{\theta}_{0}-\left(a_{1} c \beta+\lambda_{1}^{2}\right) \hat{\eta}_{0}\right] \\
c_{2}= & \frac{-1}{\left(\lambda_{2}-\lambda_{1}\right)\left(\lambda_{3}-\lambda_{2}\right)\left(\lambda_{4}-\lambda_{2}\right)}\left[-a_{1} \tilde{m} \hat{e}_{0}+\lambda_{2} \tilde{m} \hat{v}_{0}\right. \\
& \left.+\left(\tilde{k} \lambda_{2}+\lambda_{1} \lambda_{3} \lambda_{4}+\tilde{m} \tau\right) \hat{\theta}_{0}-\left(a_{1} c \beta+\lambda_{2}^{2}\right) \hat{\eta}_{0}\right]
\end{aligned}
$$




$$
\begin{aligned}
c_{3}= & \frac{1}{\left(\lambda_{3}-\lambda_{1}\right)\left(\lambda_{3}-\lambda_{2}\right)\left(\lambda_{4}-\lambda_{3}\right)}\left[-a_{1} \tilde{m} \hat{e}_{0}+\lambda_{3} \tilde{m} \hat{v}_{0}\right. \\
& \left.+\left(\tilde{k} \lambda_{3}+\lambda_{1} \lambda_{2} \lambda_{4}+\tilde{m} \tau\right) \hat{\theta}_{0}-\left(a_{1} c \beta+\lambda_{3}^{2}\right) \hat{\eta}_{0}\right], \\
c_{4}= & \frac{-1}{\left(\lambda_{4}-\lambda_{1}\right)\left(\lambda_{4}-\lambda_{2}\right)\left(\lambda_{4}-\lambda_{3}\right)}\left[-a_{1} \tilde{m} \hat{e}_{0}+\lambda_{4} \tilde{m} \hat{v}_{0}\right. \\
& \left.+\left(\tilde{k} \lambda_{4}+\lambda_{1} \lambda_{2} \lambda_{3}+\tilde{m} \tau\right) \hat{\theta}_{0}-\left(a_{1} c \beta+\lambda_{4}^{2}\right) \hat{\eta}_{0}\right] .
\end{aligned}
$$

Puting $f=0$ in formula (7), from the definition of the function $e$ we get

$$
\begin{aligned}
H & :=\xi \times \hat{u}=\cos \sqrt{a_{2}} t\left(\xi \times \widehat{u^{0}}\right)+\frac{\sin \sqrt{a_{2}} t}{\sqrt{a_{2}}}\left(\xi \times \widehat{u^{1}}\right), \\
H_{4} & :=\xi \cdot \hat{u}=-i \hat{e} .
\end{aligned}
$$

Then $\hat{u}$ can be expressed as follows:

$$
\hat{u}=\frac{H \times \xi+H_{4} \cdot \xi}{|\xi|^{2}} .
$$

Hence for $f=0, s=0$ we obtain

$$
\begin{aligned}
\hat{u} & =\left(\cos \sqrt{a_{2}} t I-\frac{\xi \otimes \xi}{|\xi|^{2}} \cos \sqrt{a_{2}} t+h_{1} \frac{\xi \otimes \xi}{|\xi|^{2}}\right) \widehat{u^{0}} \\
& +\left(\frac{\sin \sqrt{a_{2}} t}{\sqrt{a_{2}}} I-\frac{\sin \sqrt{a_{2}} t}{\sqrt{a_{2}}} \frac{\xi \otimes \xi}{|\xi|^{2}}+h_{2} \frac{\xi \otimes \xi}{|\xi|^{2}}\right) \widehat{u^{1}}+\frac{h_{3}}{|\xi|^{2}} \xi \hat{\theta}_{0}+\frac{h_{4}}{|\xi|^{2}} \xi \hat{\theta}_{1}, \\
\hat{\theta} & =\tilde{h}_{1} \widehat{\xi u^{0}}+\tilde{h}_{2} \xi \widehat{u^{1}}+\tilde{h}_{3} \hat{\theta}_{0}+\tilde{h}_{4} \hat{\theta}_{1},
\end{aligned}
$$

where

$$
\begin{aligned}
h_{1}= & -\frac{a_{1} \tilde{m}}{\left(\lambda_{2}-\lambda_{1}\right)\left(\lambda_{3}-\lambda_{1}\right)\left(\lambda_{4}-\lambda_{1}\right)} Y_{11}+\frac{a_{1} \tilde{m}}{\left(\lambda_{2}-\lambda_{1}\right)\left(\lambda_{3}-\lambda_{2}\right)\left(\lambda_{4}-\lambda_{2}\right)} Y_{21} \\
& -\frac{a_{1} \tilde{m}}{\left(\lambda_{3}-\lambda_{1}\right)\left(\lambda_{3}-\lambda_{2}\right)\left(\lambda_{4}-\lambda_{3}\right)} Y_{31}+\frac{a_{1} \tilde{m}}{\left(\lambda_{4}-\lambda_{1}\right)\left(\lambda_{4}-\lambda_{2}\right)\left(\lambda_{4}-\lambda_{3}\right)} Y_{41}, \\
h_{2}= & \frac{\lambda_{1} \tilde{m}}{\left(\lambda_{2}-\lambda_{1}\right)\left(\lambda_{3}-\lambda_{1}\right)\left(\lambda_{4}-\lambda_{1}\right)} Y_{11}-\frac{\lambda_{2}}{\left(\lambda_{2}-\lambda_{1}\right)\left(\lambda_{3}-\lambda_{2}\right)\left(\lambda_{4}-\lambda_{2}\right)} Y_{21} \\
& +\frac{\lambda_{3} \tilde{m}}{\left(\lambda_{3}-\lambda_{1}\right)\left(\lambda_{3}-\lambda_{2}\right)\left(\lambda_{4}-\lambda_{3}\right)} Y_{31}-\frac{\lambda_{4} \tilde{m}}{\left(\lambda_{4}-\lambda_{1}\right)\left(\lambda_{4}-\lambda_{2}\right)\left(\lambda_{4}-\lambda_{3}\right)} Y_{41}, \\
h_{3}= & -i\left[\frac{\tilde{k} \lambda_{1}+\lambda_{2} \lambda_{3} \lambda_{4}+\tilde{m} \tau}{\left(\lambda_{2}-\lambda_{1}\right)\left(\lambda_{3}-\lambda_{1}\right)\left(\lambda_{4}-\lambda_{1}\right)} Y_{11}-\frac{\tilde{k} \lambda_{2}+\lambda_{1} \lambda_{3} \lambda_{4}+\tilde{m} \tau}{\left(\lambda_{2}-\lambda_{1}\right)\left(\lambda_{3}-\lambda_{2}\right)\left(\lambda_{4}-\lambda_{2}\right)} Y_{21}\right. \\
& \left.+\frac{\tilde{k} \lambda_{3}+\lambda_{1} \lambda_{2} \lambda_{4}+\tilde{m} \tau}{\left(\lambda_{3}-\lambda_{1}\right)\left(\lambda_{3}-\lambda_{2}\right)\left(\lambda_{4}-\lambda_{3}\right)} Y_{31}-\frac{\tilde{k} \lambda_{4}+\lambda_{1} \lambda_{2} \lambda_{3}+\tilde{m} \tau}{\left(\lambda_{4}-\lambda_{1}\right)\left(\lambda_{4}-\lambda_{2}\right)\left(\lambda_{4}-\lambda_{3}\right)} Y_{41}\right], \\
h_{4}= & -i\left[-\frac{a_{1} c \beta+\lambda_{1}^{2}}{\left(\lambda_{2}-\lambda_{1}\right)\left(\lambda_{3}-\lambda_{1}\right)\left(\lambda_{4}-\lambda_{1}\right)} Y_{11}+\frac{a_{1} c \beta}{\left(\lambda_{2}-\lambda_{1}\right)\left(\lambda_{3}-\lambda_{2}\right)\left(\lambda_{4}-\lambda_{2}\right)} Y_{21}\right. \\
& \left.-\frac{a_{1} c \beta+\lambda_{3}^{2}}{\left(\lambda_{3}-\lambda_{1}\right)\left(\lambda_{3}-\lambda_{2}\right)\left(\lambda_{4}-\lambda_{3}\right)} Y_{31}+\frac{a_{1} c \beta+\lambda_{4}^{2}}{\left(\lambda_{4}-\lambda_{1}\right)\left(\lambda_{4}-\lambda_{2}\right)\left(\lambda_{4}-\lambda_{3}\right)} Y_{41}\right],
\end{aligned}
$$




$$
\begin{aligned}
\tilde{h}_{1}= & -i\left[\frac{a_{1} \tilde{m}}{\left(\lambda_{2}-\lambda_{1}\right)\left(\lambda_{3}-\lambda_{1}\right)\left(\lambda_{4}-\lambda_{1}\right)} e^{\lambda_{1} t}+\frac{a_{1} \tilde{m}}{\left(\lambda_{2}-\lambda_{1}\right)\left(\lambda_{3}-\lambda_{2}\right)\left(\lambda_{4}-\lambda_{2}\right)} e^{\lambda_{2} t}\right. \\
& \left.-\frac{a_{1} \tilde{m}}{\left(\lambda_{3}-\lambda_{1}\right)\left(\lambda_{3}-\lambda_{2}\right)\left(\lambda_{4}-\lambda_{3}\right)} e^{\lambda_{3} t}+\frac{a_{1}}{\left(\lambda_{4}-\lambda_{1}\right)\left(\lambda_{4}-\lambda_{2}\right)\left(\lambda_{4}-\lambda_{3}\right)} e^{\lambda_{4} t}\right], \\
\tilde{h}_{2}= & i\left[\frac{\lambda_{2} \tilde{m}}{\left(\lambda_{2}-\lambda_{1}\right)\left(\lambda_{3}-\lambda_{1}\right)\left(\lambda_{4}-\lambda_{1}\right)} e^{\lambda_{1} t}-\frac{\lambda_{4} \tilde{m}}{\left(\lambda_{2}-\lambda_{1}\right)\left(\lambda_{3}-\lambda_{2}\right)\left(\lambda_{4}-\lambda_{2}\right)} e^{\lambda_{2} t}\right. \\
& +\frac{\lambda_{3} \tilde{m}}{\left(\lambda_{3}-\lambda_{1}\right)\left(\lambda_{3}-\lambda_{2}\right)\left(\lambda_{4}-\lambda_{3}\right)} e^{\lambda_{3} t}-\frac{\left.\lambda_{4}-\lambda_{1}\right)\left(\lambda_{4}-\lambda_{2}\right)\left(\lambda_{4}-\lambda_{3}\right)}{\left(\lambda_{4}\right)} e^{\lambda_{4} t}, \\
\tilde{h}_{3}= & \frac{\tilde{k} \lambda_{1}+\lambda_{2} \lambda_{3} \lambda_{4}+\tilde{m} \tau}{\left(\lambda_{2}-\lambda_{1}\right)\left(\lambda_{3}-\lambda_{1}\right)\left(\lambda_{4}-\lambda_{1}\right)} e^{\lambda_{1} t}+\frac{\tilde{k} \lambda_{2}+\lambda_{1} \lambda_{3} \lambda_{4}+\tilde{m} \tau}{\left(\lambda_{2}-\lambda_{1}\right)\left(\lambda_{3}-\lambda_{2}\right)\left(\lambda_{4}-\lambda_{2}\right)} e^{\lambda_{2} t} \\
& +\frac{\tilde{k} \lambda_{3}+\lambda_{1} \lambda_{2} \lambda_{4}+\tilde{m} \tau}{\left(\lambda_{3}-\lambda_{1}\right)\left(\lambda_{3}-\lambda_{2}\right)\left(\lambda_{4}-\lambda_{3}\right)} e^{\lambda_{3} t}-\frac{\tilde{k} \lambda_{4}+\lambda_{1} \lambda_{2} \lambda_{3}+\tilde{m} \tau}{\left(\lambda_{4}-\lambda_{1}\right)\left(\lambda_{4}-\lambda_{2}\right)\left(\lambda_{4}-\lambda_{3}\right)} e^{\lambda_{4} t}, \\
\tilde{h}_{4}= & \frac{a_{1} c \beta+\lambda_{1}^{2}}{\left(\lambda_{2}-\lambda_{1}\right)\left(\lambda_{3}-\lambda_{1}\right)\left(\lambda_{4}-\lambda_{1}\right)} e^{\lambda_{1} t}-\frac{a_{1} c \beta+\lambda_{2}^{2}}{\left(\lambda_{2}-\lambda_{1}\right)\left(\lambda_{3}-\lambda_{2}\right)\left(\lambda_{4}-\lambda_{2}\right)} e^{\lambda_{2} t} \\
& +\frac{a_{1} c \beta+\lambda_{3}^{2}}{\left(\lambda_{3}-\lambda_{1}\right)\left(\lambda_{3}-\lambda_{2}\right)\left(\lambda_{4}-\lambda_{3}\right)} e^{\lambda_{3} t}-\frac{a_{1} c \beta+\lambda_{4}^{2}}{\left(\lambda_{4}-\lambda_{1}\right)\left(\lambda_{4}-\lambda_{2}\right)\left(\lambda_{4}-\lambda_{3}\right)} e^{\lambda_{4} t} .
\end{aligned}
$$

Now we construct a solution of the initial value problem (1) with null Cauchy data. We know that (cf. (7))

$$
\hat{w}=\int_{0}^{t} \frac{\sin \left[\sqrt{a_{2}}(t-s)\right] \widehat{\operatorname{rot} f}}{\sqrt{a_{2}}} d s .
$$

To find the fundamental solution of (8) one has to solve the problem

$$
\mathbb{Y}^{\prime}=A \mathbb{Y}, \quad \mathbb{Y}(0)=I
$$

where $\mathbb{Y}=\left\{H_{i j}\right\}$ is a matrix with columns $\mathbb{Y}_{1}, \mathbb{Y}_{2}, \mathbb{Y}_{3}$. After a simple calculation we get

$$
\begin{aligned}
\mathbb{Y}_{1}= & \frac{-a_{1} \tilde{m}}{\left(\lambda_{2}-\lambda_{1}\right)\left(\lambda_{3}-\lambda_{1}\right)\left(\lambda_{4}-\lambda_{1}\right)} Y_{1}+\frac{a_{1} \tilde{m}}{\left(\lambda_{2}-\lambda_{1}\right)\left(\lambda_{3}-\lambda_{2}\right)\left(\lambda_{4}-\lambda_{2}\right)} Y_{2} \\
& -\frac{a_{1} \tilde{m}}{\left(\lambda_{3}-\lambda_{1}\right)\left(\lambda_{3}-\lambda_{2}\right)\left(\lambda_{4}-\lambda_{3}\right)} Y_{3}+\frac{a_{1}}{\left(\lambda_{4}-\lambda_{1}\right)\left(\lambda_{4}-\lambda_{2}\right)\left(\lambda_{4}-\lambda_{3}\right)} Y_{4}, \\
\mathbb{Y}_{2}= & \frac{\lambda_{1} \tilde{m}}{\left(\lambda_{2}-\lambda_{1}\right)\left(\lambda_{3}-\lambda_{1}\right)\left(\lambda_{4}-\lambda_{1}\right)} Y_{1}-\frac{\lambda_{2} \tilde{m}}{\left(\lambda_{2}-\lambda_{1}\right)\left(\lambda_{3}-\lambda_{2}\right)\left(\lambda_{4}-\lambda_{2}\right)} Y_{2} \\
& -\frac{\lambda_{3} \tilde{m}}{\left(\lambda_{3}-\lambda_{1}\right)\left(\lambda_{3}-\lambda_{2}\right)\left(\lambda_{4}-\lambda_{3}\right)} Y_{3}-\frac{\lambda_{4}}{\left(\lambda_{4}-\lambda_{1}\right)\left(\lambda_{4}-\lambda_{2}\right)\left(\lambda_{4}-\lambda_{3}\right)} Y_{4}, \\
\mathbb{Y}_{3}= & \frac{\tilde{k} \lambda_{1}+\lambda_{2} \lambda_{3} \lambda_{4}+\tau \tilde{m}}{\left(\lambda_{2}-\lambda_{1}\right)\left(\lambda_{3}-\lambda_{1}\right)\left(\lambda_{4}-\lambda_{1}\right)} Y_{1}-\frac{\tilde{k} \lambda_{2}+\lambda_{1} \lambda_{3} \lambda_{4}+\tilde{m} \tau}{\left(\lambda_{2}-\lambda_{1}\right)\left(\lambda_{3}-\lambda_{2}\right)\left(\lambda_{4}-\lambda_{2}\right)} Y_{2} \\
& -\frac{\tilde{k} \lambda_{3}+\lambda_{1} \lambda_{2} \lambda_{4}+\tilde{m} \tau}{\left(\lambda_{3}-\lambda_{1}\right)\left(\lambda_{3}-\lambda_{2}\right)\left(\lambda_{4}-\lambda_{3}\right)} Y_{3}-\frac{\tilde{k} \lambda_{4}+\lambda_{1} \lambda_{2} \lambda_{3}+\tilde{m} \tau}{\left(\lambda_{4}-\lambda_{1}\right)\left(\lambda_{4}-\lambda_{2}\right)\left(\lambda_{4}-\lambda_{3}\right)} Y_{4},
\end{aligned}
$$




$$
\begin{aligned}
\mathbb{Y}_{4}= & -\frac{a_{1} c \beta+\lambda_{1}^{2}}{\left(\lambda_{2}-\lambda_{1}\right)\left(\lambda_{3}-\lambda_{1}\right)\left(\lambda_{4}-\lambda_{1}\right)} Y_{1}+\frac{a_{1} c \beta+\lambda_{2}^{2}}{\left(\lambda_{2}-\lambda_{1}\right)\left(\lambda_{3}-\lambda_{2}\right)\left(\lambda_{4}-\lambda_{2}\right)} Y_{2} \\
& -\frac{a_{1} c \beta+\lambda_{3}^{2}}{\left(\lambda_{3}-\lambda_{1}\right)\left(\lambda_{3}-\lambda_{2}\right)\left(\lambda_{4}-\lambda_{3}\right)} Y_{3}+\frac{a_{1} c \beta+\lambda_{4}^{2}}{\left(\lambda_{4}-\lambda_{1}\right)\left(\lambda_{4}-\lambda_{2}\right)\left(\lambda_{4}-\lambda_{3}\right)} Y_{4} .
\end{aligned}
$$

Therefore the fundamental solution is equal to $\mathbb{H}=h(t)\left\{H_{i j}\right\}$, where $h$ denotes the Heaviside function. The solution of (8) with $G=[0, \widehat{\operatorname{div} f}, 0, \hat{\mathbf{s}}]^{T}$ is

$$
Y=\int_{0}^{t}\left\{H_{i j}(t-s)\right\} G(s) d s .
$$

Let $-\hat{w}=S=\left[S_{1}, S_{2}, S_{3}\right]^{T}, S_{4}=-i \hat{e}$. We have

$$
\hat{u}=\frac{\xi S_{4}}{|\xi|^{2}}+\frac{S \times \xi}{|\xi|^{2}}
$$

and consequently

$$
\begin{aligned}
\hat{u}= & \int_{0}^{t}\left\{\frac{\sin \left[\sqrt{a_{2}}(t-s)\right]}{\sqrt{a_{2}}} I-\frac{\xi \otimes \xi \sin \left[\sqrt{a_{2}}(t-s)\right]}{|\xi|^{2}}+\frac{\xi \otimes \xi}{\sqrt{a_{2}}} H_{12}(t-s)\right\} \hat{f} d s \\
& +\int_{0}^{t}\left[H_{14}(t-s)(-i) \xi \hat{\mathbf{s}}\right] d s, \\
\hat{\theta}= & \int_{0}^{t}\left[H_{32}(t-s) i \xi \hat{f}+H_{34}(t-s) \hat{\mathbf{s}}\right] d s .
\end{aligned}
$$

The solution of the problem (1-2) is of course the sum of the solutions (12) and (13).

For further investigation of this solution we derive the asymptotic behaviour of the roots $\lambda_{1}, \lambda_{2}, \lambda_{3}, \lambda_{4}$. For $|\xi| \rightarrow 0$ we get

$$
\begin{aligned}
\lambda_{1} & =\frac{-k c_{1}^{2}}{c c_{1}^{2}+b}|\xi|^{2}+o\left(|\xi|^{2}\right), \quad \text { where } \quad b=\frac{T_{0} m^{2}}{\rho}, \\
\lambda_{2} & =-\frac{1}{\beta}+q|\xi|^{2}+o\left(|\xi|^{2}\right), \quad \text { where } \quad q=\mathrm{const}, \\
\lambda_{3 / 4} & = \pm \sqrt{\frac{k}{c \beta}}|\xi|+o(|\xi|),
\end{aligned}
$$

and for $|\xi| \rightarrow \infty$,

$$
\lambda_{1 / 2}= \pm i \sqrt{\frac{k}{c \beta}}|\xi|+O(1), \quad \lambda_{3 / 4}= \pm i c_{1} l_{1}|\xi|^{2}+O(|\xi|) .
$$

Proof of Theorem 1. Let $\langle\xi\rangle=\sqrt{1+|\xi|^{2}},\|\cdot\|_{L_{2}}=\|\cdot\|$ and let $C_{1}$ denote a large constant. Note that $h_{1 \mid t=0}=1$, and in view of the asymptotic 
behaviour and the properties of $\lambda_{1}, \lambda_{2}, \lambda_{3}, \lambda_{4}$, the function $h_{1}$ is bounded. Hence

$$
\begin{aligned}
\int_{\mathbb{R}^{3}}\left|\left(\cos \sqrt{a_{2}} t I-\frac{\xi \otimes \xi}{|\xi|^{2}} \cos \sqrt{a_{2}} t+h_{1} \frac{\xi \otimes \xi}{|\xi|^{2}}\right) \widehat{u^{0}}\right|^{2}\langle\xi\rangle^{2 s} d \xi & \\
& \leq C_{1} \int\langle\xi\rangle^{2 s}\left|\widehat{u^{0}}\right|^{2} d \xi=C_{1}\left\|u^{0}\right\|_{s}^{2} .
\end{aligned}
$$

Note that $h_{2 \mid t=0}=0, h_{2}=h_{2}^{\prime}(\theta t) t, \theta \in(0,1)$, and hence

$$
\begin{aligned}
\int_{\mathbb{R}^{3}}\left|\frac{\sin \sqrt{a_{2}} t}{\sqrt{a^{2}}} I-\frac{\sin \sqrt{a_{2}} t}{\sqrt{a_{2}}} \frac{\xi \otimes \xi}{|\xi|^{2}}+h_{2} \frac{\xi \otimes \xi}{\left.|\xi|^{2}\right|^{2}}\right|^{2}\left|\widehat{u^{1}}\right|^{2}\langle\xi\rangle^{2 s} d \xi \\
\leq C_{1}\left(1+t^{2}\right) \int_{\mathbb{R}^{3}}\langle\xi\rangle^{2 s}\left|\widehat{u^{1}}\right|^{2} d \xi=C_{1}\left(1+t^{2}\right)\left\|u^{1}\right\|_{s}^{2} .
\end{aligned}
$$

Now $h_{3 \mid t=0}=h_{4 \mid t=0}=0$, so

$$
\begin{gathered}
\int_{\mathbb{R}^{3}}\left|\frac{h_{3}}{|\xi|^{2}} \xi \hat{\theta}_{0}\right|^{2}\langle\xi\rangle^{2 s} d \xi \leq C_{1}\left(1+t^{2}\right) \int_{\mathbb{R}^{3}}\left|\hat{\theta}_{0}\right|\langle\xi\rangle^{2 s+2} d \xi=C_{1}\left(1+t^{2}\right)\left\|\theta_{0}\right\|_{s+1}^{2}, \\
\int_{\mathbb{R}^{3}}\left|\frac{h_{4}}{|\xi|^{2}} \xi \hat{\theta}_{1}\right|^{2}\langle\xi\rangle^{2 s} d \xi \leq C_{1}\left(1+t^{2}\right)\left\|\theta_{1}\right\|_{s+1}^{2} .
\end{gathered}
$$

Next, $\tilde{h}_{1 \mid t=0}=0$, and therefore

$$
\int_{\mathbb{R}^{3}}\left|\tilde{h}_{1} \widehat{\xi u^{0}}\right|^{2}\langle\xi\rangle^{2 s} d \xi \leq C_{1}\left(1+t^{2}\right)\left\|u^{0}\right\|_{s+1}^{2} .
$$

Moreover $\tilde{h}_{2 \mid t=0}=0,\left|\tilde{h}_{2}\right| \leq d /\langle\xi\rangle^{2}$, where $d=$ const, so

$$
\int_{\mathbb{R}^{3}}\left|\tilde{h}_{2} \xi \widehat{u^{1}}\right|^{2}\langle\xi\rangle^{2 s} d \xi \leq C_{1}\left(1+t^{2}\right)\left\|u^{0}\right\|_{s-1}^{2} .
$$

Furthermore $\tilde{h}_{3 \mid t=0}=1, \tilde{h}_{4 \mid t=0}=0$, so

$$
\begin{aligned}
& \int_{\mathbb{R}^{3}}\left|\tilde{h}_{3} \hat{\theta}_{0}\right|^{2}\langle\xi\rangle^{2 s} d \xi \leq C_{1}\left\|\theta_{0}\right\|_{s}^{2}, \\
& \int_{\mathbb{R}^{3}}\left|\tilde{h}_{4} \hat{\theta}_{1}\right|^{2}\langle\xi\rangle^{2 s} d \xi \leq C_{1}\left(1+t^{2}\right)\left\|\theta_{1}\right\|_{s}^{2} .
\end{aligned}
$$

Since $H_{12 \mid t=0}=H_{14 \mid t=0}=H_{32 \mid t=0}=H_{34 \mid t=0}=0$ we have

$$
\begin{aligned}
\int_{\mathbb{R}^{3}} \mid \int_{0}^{t}\left\{\frac{\sin \left[\sqrt{a_{2}}(t-s)\right]}{\sqrt{a_{2}}} I-\frac{\xi \otimes \xi \sin \left[\sqrt{a_{2}}(t-s)\right]}{|\xi|^{2}}\right. & \left.+\frac{\xi \otimes \xi}{\sqrt{a_{2}}} H_{12}(t-s)\right\}\left.\hat{f} d s\right|^{2}\langle\xi\rangle^{2 s} d \xi \\
& \leq C_{1}\left(1+t^{4}\right) \sup _{\tau \in[0, t]}\|f(\tau)\|_{s}^{2},
\end{aligned}
$$




$$
\begin{gathered}
\int_{\mathbb{R}^{3}}\left|\int_{0}^{t} H_{14}(t-s)(-i) \xi \hat{\mathbf{s}} d s\right|^{2}\langle\xi\rangle^{2 s} d \xi \leq C_{1}\left(1+t^{4}\right) \sup _{\tau \in[0, t]}\|\mathbf{s}\|_{s+1}^{2}, \\
\quad \int_{\mathbb{R}^{3}}\left|\int_{0}^{t} H_{32}(t-s) i \xi \hat{f} d s\right|^{2}\langle\xi\rangle^{2 s} d \xi \leq C_{1}\left(1+t^{4}\right) \sup _{\tau \in[0, t]}\|f\|_{s-1}^{2}, \\
\int_{\mathbb{R}^{3}}\left|\int_{0}^{t} H_{34}(t-s) \hat{\mathbf{s}} d s\right|^{2}\langle\xi\rangle^{2 s} d \xi \leq C_{1}\left(1+t^{4}\right) \sup _{\tau \in[0, t]}\|\mathbf{s}\|_{s+1}^{2} .
\end{gathered}
$$

The other elements of $\|u\|_{s}^{2},\|\theta\|_{s}^{2}$ can be estimated by using the inequality $a b \leq \frac{1}{2}\left(a^{2}+b^{2}\right)$.

\section{References}

[1] G. Ahmadi and K. Firoozbakhsh, First strain-gradient theory of thermoelasticity, Int. J. Solids Structures 11 (1975), 339-345.

[2] A. E. Green and N. Laws, On the entropy production inequality, Arch. Ration. Mech. Anal. 45 (1972), 47-59.

[3] D. Iesan, Thermoelasticity of nonsimple materials, J. Thermal Stresses 6 (1983), $167-188$.

[4] H. Kołakowski and J. Łazuka, The Cauchy problem for the system of partial differential equations describing nonsimple thermoelasticity, Appl. Math. (Warsaw) 35 (2008), 97-105.

[5] L. S. Pontryagin, Ordinary Differential Equations, Addison-Wesley, 1962.

Henryk Kołakowski, Jarosław Łazuka

Institute of Mathematics and Cryptology

Faculty of Cybernetics

Military University of Technology

S. Kaliskiego 2

01-489 Warszawa, Poland

E-mail: jkolakowski@wat.edu.pl jlazuka@wat.edu.pl 